Revista Brasileira de Agricultura Irrigada v.9, nº.6, p 383 - 391, 2015

ISSN 1982-7679 (On-line)

Fortaleza, CE, INOVAGRI - http://www.inovagri.org.br

DOI: $10.7127 /$ rbai.v9n600346

Protocolo 346.15 - 14/07/2015 Aprovado em 10/11/2015

\title{
DESEMPENHO HIDRÁULICO E PERFIL DE DISTRIBUIÇÃO DE ÁGUA DE EMISSORES TIPO MICROASPERSOR MULTIFUNCIONAL
}

\author{
Miguel Julio Machado Guimarães ${ }^{1}$, Hammady Ramalho Soares ${ }^{1}$, Pedro Robinson Fernandes \\ de Medeiros ${ }^{2}$, Enio Farias de França e Silva ${ }^{3}$
}

\begin{abstract}
RESUMO
Objetivou-se com o presente trabalho objetivou avaliar as características hidráulicas e a uniformidade de distribuição de água a partir de coeficientes de uniformidades apresentados na literatura (CUC, CUD e CUE) de emissores microaspesores tipo multifuncional. Os ensaios foram realizados na área experimental do Departamento de Tecnologia Rural UFRPE. Foram avaliados microaspesores MFN com bailarina preta e bocais de cor grafite ( $(=0,80 \mathrm{~mm})$ e laranja $(\mathscr{\varnothing}=1,80 \mathrm{~mm})$. Para determinação da vazão, cada emissor foi submetido às pressões de 250; 200; 150; 100 e $50 \mathrm{kPa}$. As vazões foram coletadas em um período de seis minutos (10\% da vazão média) e medidas utilizando uma balança de precisão. Para avaliar a distribuição foram utilizados coletores com área de $50 \mathrm{~cm}^{2}$, dispostos nas linhas dos quatros quadrantes, espaçados em $0,3 \mathrm{~m}$. O coeficiente de variação de fabricação médio para os bocais de cor grafite e laranja foram de 1,66\% e 5,07\%, respectivamente, sendo classificados como boa uniformidade, segundo a ABNT. Quanto à vazão, verificou-se que os ensaios realizados apresentaram uma pequena tendência à superestimativa, em torno de 8,02\% em média. Apesar de todas as configurações de sobreposições testadas apresentarem bons resultados de CUC, CUD e CUE, apenas o bocal grafite obteve resultados com o diâmetro próximo ao fornecido pelo fabricante.
\end{abstract}

Palavras-chave: Irrigação localizada, uniformidade, vazão média.

\section{HYDRAULIC PERFORMANCE AND PROFILE OF WATER DISTRIBUTION OF EMITTERS MULTIFUNCTIONAL MICROSPRINKLER}

\footnotetext{
ABSTRACT

${ }^{1}$ Departamento de Engenharia Agrícola - Universidade Federal Rural de Pernambuco, Rua D Manoel Medeiros, s/n, Dois Irmãos, Recife/PE. E-mail: mjmguimaraes@hotmail.com/hresoares@hotmail.com

${ }^{2}$ Colegiado de Engenharia Agrícola e Ambiental - Universidade Federal do Vale do São Francisco, Campus Juazeiro, Avenida Antônio Carlos Magalhães, 510 - Santo Antônio, Juazeiro-BA. Email: prfmede@hotmail.com

${ }^{3}$ Programa de Pós-Graduação em Engenharia Agrícola - Universidade Federal Rural de Pernambuco, Rua D Manoel Medeiros, s/n, Dois Irmãos, Recife/PE. Email: enio.silva@deagri.ufrpe.br
} 
This study aimed to evaluate the hydraulic characteristics and uniformity of water distribution from the uniformity coefficients (CUC, CUE and CUD) of emitters microsprinkler type multifunctional. The tests were carried out in the experimental area of the Department of Rural Technology - UFRPE. It were evaluated microsprinkler MFN with black ballerina and nozzles with colors graphite $(\dot{\varnothing}=0.80 \mathrm{~mm})$ and orange $(\dot{\varnothing}=1.80 \mathrm{~mm})$. For the determination the flow rate, each emitter was subjected to pressures of 250, 200, 150, 100 and $50 \mathrm{kPa}$. Flow rates were collected a period of six minutes (10\% of average flow) and measured using a precision balance. To evaluate the distribution collectors were used with area $50 \mathrm{~cm}^{2}$, arranged in rows of four quadrants spaced $0.3 \mathrm{~m}$. The coefficient of manufacturing average variation for the nozzle of colors graphite and orange were $1.66 \%$ and $5.07 \%$, respectively, classified as good uniformity, according to ABNT. As for the flow, it was verified that tests showed a small tendency to overestimate, around $8.02 \%$ on average. Despite all configurations tested overlays have presented good results of CUC, CUE and CUD, only the graphite nozzle obtained results with diameter next to that provided by the manufacturer.

Keywords: Trickle irrigation, uniformity, average flow.

\section{INTRODUÇÃO}

A necessidade de conservação dos recursos hídricos e redução nos custos de produção, principalmente de energia e de insumos, devem, por meio dos sistemas de irrigação e manejo, proporcionar aplicação de água uniforme e eficiente (REZENDE et al., 2002). No entanto, para que a irrigação seja eficiente, é imperativo que os sistemas apresentem alta uniformidade de aplicação da água. Uma vez instalado o projeto de irrigação, é necessário verificar se as condições previstas inicialmente se confirmam em campo, para tanto, deve-se avaliar as condições de pressão, vazão e lâminas d'água aplicadas.

Altos valores de uniformidade de aplicação de água são fundamentais para a obtenção de elevadas produtividades de uma cultura com um menor consumo de água, energia e fertilizantes (ÁLVAREZ et al., 2004). De acordo com Louie e Selker (2000) devido a grande necessidade de conservação dos recursos hídricos, é crescente a preocupação com a uniformidade de aplicação de água pelos fabricantes de microaspersores, projetistas e usuários de sistemas de irrigação.

Segundo Prado e Colombo (2009), a existência de inúmeras possibilidades de combinação de pressão de serviço e diâmetro de bocais condicionam os fabricantes a não disponibilizarem ou a não apresentarem, de maneira adequada, as características técnicas de distribuição de água de seus microaspersores.

Por outro lado, o emissor é a parte chave no sistema de irrigação localizada, projetado para permitir que a água, sob pressão no tubo, produza uma vazão de maneira uniforme, com diâmetros próximos a $0,51 \mathrm{~mm}$ no geral; sendo de fácil obstrução a partir de partículas físicas, químicas e biológicas (DETOMINI et al., 2009).

Emissores do tipo microaspersores proporcionam maior superfície de solo molhado em relação ao gotejamento, a um menor custo fixo. Em solos arenosos e na irrigação de cultivos arbóreos a utilização de microaspersores, em geral, é mais vantajosa que a utilização de gotejadores, além do que são menos susceptíveis à obstrução e menos exigentes em filtragem da água (FRIZZONE \& DOURADO NETO, 2003; MARQUES et al., 2006; MACEDO et al., 2010), este tipo de emissor opera a pressões e vazões de 100 a $200 \mathrm{kPa}$ e 30 a $200 \mathrm{~L} \mathrm{~h}^{-1}$, respectivamente; nos sistemas regulados, o consumo de energia é aumentado e são susceptíveis à deriva e evaporação.

Quanto à seleção desse tipo de emissor, podemos citar alguns fatores intervenientes como o tipo de solo a ser irrigado, necessidades de água das plantas, vazão do emissor, qualidade da água, vazão disponível, condições de vento e demanda evaporativa da 


\section{DESEMPENHO HIDRÁULICO E PERFIL DE DISTRIBUIÇÃO DE ÁGUA DE EMISSORES TIPO MICROASPERSOR MULTIFUNCIONAL}

atmosfera, características topográficas do terreno, custos do emissor e riscos inerentes ao sistema, porcentagem de área molhada (SOUZA, 2005). Geralmente os emissores que oferecem as características mais desejáveis e oferecem os menores riscos ao sistema são mais caros (RETTORE NETO et al., 2009).

Emissores são dispositivos com descarga através de pequenas seções; 0 seu desempenho hidráulico foi caracterizado por uma função potencial empírica que descreve as características de vazão (q) versus pressão do emissor $(H)$, cuja vazão tem relação direta com a carga hidráulica atuante na entrada do emissor (KELLER; KARMELI, 1975; SOUZA, 2005).

Com isso, objetivou-se com o presente trabalho avaliar emissores do tipo microaspersores disponíveis no mercado para o pequeno produtor quanto às suas características hidráulicas e uniformidade de aplicação de água.

\section{MATERIAL E MÉTODOS}

Os ensaios foram realizados no Laboratório de Água e Solo no Departamento de Engenharia Agrícola da Universidade Federal Rural de Pernambuco - UFRPE no ano de 2013. Foram avaliados emissores do tipo microaspersores MFN com bailarina preta, nos bocais de cor grafite ( $\varnothing=0,80 \mathrm{~mm}$ ) e laranja (ஜ́=1,80mm) (Tabela 1$)$.

Tabela 1. Características técnicas dos emissores

\begin{tabular}{|c|c|c|c|c|c|c|c|}
\hline \multirow{3}{*}{ Cor do Bocal } & \multirow{3}{*}{$\begin{array}{l}\text { Diâmetro } \\
(\mathscr{\varnothing}-\mathrm{mm})\end{array}$} & \multirow{3}{*}{$\begin{array}{l}\text { Diâmetro Molhado* } \\
\text { (ஜ́ - m) }\end{array}$} & \multicolumn{5}{|c|}{ Vazão $\left(\mathrm{L} \mathrm{h} \mathrm{h}^{-1}\right)$} \\
\hline & & & \multicolumn{5}{|c|}{ Pressão (kPa) } \\
\hline & & & $50 * *$ & 100 & 150 & 200 & 250 \\
\hline Grafite & 0,80 & 5,50 & - & 25 & 31 & 36 & 41 \\
\hline Laranja & 1,80 & 7,00 & - & 91 & 112 & 129 & 145 \\
\hline
\end{tabular}

*Diâmetro molhado médio medido à pressão nominal $(150 \mathrm{kPa})$; ** Dados não fornecidos pelo fabricante. Fonte: Catálogo Fabricante.

Foi utilizada uma estrutura composta por uma caixa d’água de PVC com capacidade de $0,5 \mathrm{~m}^{3}$, acoplada a uma bomba com pressão máxima de $400 \mathrm{kPa}$. Os emissores foram fixados com microtubo $(0,4 \mathrm{~cm}$ de diâmetro por $50 \mathrm{~cm}$ de comprimento) a um tubo de polietileno ( $́=16 \mathrm{~mm}$ ). A pressão foi controlada por um registro de gaveta de 3/4" e medida utilizando um manômetro do tipo Bourdon.

Para determinação da vazão, cada emissor foi submetido às seguintes pressões 250; 200; 150; 100 e $50 \mathrm{kPa}$, sendo colocado sobre o emissor um recipiente capaz de coletar toda a água emitida em um período de seis minutos (10\% da vazão média), posteriormente os volumes coletados foram pesados em balança digital com precisão de três casas decimais. Os valores coletados foram convertidos em $\mathrm{L} \mathrm{h}^{-1}$ para determinação da vazão de cada peça.
Para avaliar a distribuição e uniformidade, foram utilizados coletores com área $50 \mathrm{~cm}^{2}$, dispostos em uma única linha nos quatro quadrantes, espaçados em $0,3 \mathrm{~m}$. Os volumes coletados ao final de cada ensaio foram medidos em balança digital, com precisão de três casas decimais.

Foram analisadas quatro combinações de configuração de sobreposições, 1,2 x 1,2; 1,5 x 1,5; 2,1 x 2,1 e 3,0 x 3,0 m. Para cada configuração foi determinado o Coeficiente de Uniformidade de Christiansen (CUC), o Coeficiente de Uniformidade de Distribuição (CUD) e o Coeficiente de Uniformidade Estatística (CUE) conforme as Equações 1, 2 e 3.

$$
C U C=\left(1-\frac{\sum_{i=1}^{N}\left|L_{i}-L_{m}\right|}{N L_{m}}\right) * 100
$$

em que: 
CUC = Coeficiente de Uniformidade de Christiansen, em \%;

$\mathrm{Li}=$ Vazão de cada emissor, em $\mathrm{L} \mathrm{h}^{-1}$;

$\mathrm{Lm}=$ Vazão média dos emissores, em $\mathrm{L} \mathrm{h}^{-}$ 1 ;

$\mathrm{N}=$ Número de emissores.

$C U D=\frac{100 \bar{X}_{25}}{\bar{X}}$

em que:

CUD = Coeficiente de Uniformidade de Distribuição, em \%;

$\bar{X}_{25}=$ valor médio obtido em $25 \%$ do total de pluviômetros que coletaram as

menores precipitações, em mm;

$\bar{X}=$ lâmina média observada nos pluviômetros, em mm.
$C U E=100\left(1-\frac{S_{d}}{\bar{X}}\right)$

em que:

CUE = Coeficiente de Uniformidade Estática, em \%

$\mathrm{S}_{\mathrm{d}}=$ desvio-padrão dos valores de precipitação, em mm;

$\bar{X}=$ lâmina média observada nos pluviômetros, em mm.

A interpretação destes coeficientes baseou-se na metodologia proposta por Mantovani (2001) apresentada na Tabela 2.

Tabela 2. Classificação dos valores do desempenho de sistemas de irrigação localizada em função do Coeficiente de Uniformidade de Christiansen (CUC), do Coeficiente de Uniformidade de Distribuição (CUD) e do Coeficiente de Uniformidade Estatística (CUE)

\begin{tabular}{cccc}
\hline Classe & CUC (\%) & CUD (\%) & CUE (\%) \\
\hline Excelente & $>90$ & $>84$ & $>90$ \\
Bom & $80-90$ & $68-84$ & $80-90$ \\
Razoável & $70-80$ & $52-68$ & $70-80$ \\
Ruim & $60-70$ & $36-52$ & $60-70$ \\
Inaceitável & $<60$ & $<36$ & $<60$ \\
\hline
\end{tabular}

Fonte: Mantovani (2001).

\section{RESULTADOS E DISCUSSÃO}

\section{Desempenho hidráulico}

Na Tabela 3 são apresentadas as vazões médias obtidas para cada emissor nas pressões de 50, 100, 150,
200 e $250 \mathrm{kPa}$, com os respectivos índices de variação.

Tabela 3. Valores médios de vazão $\left(\mathrm{L} \mathrm{h}^{-1}\right)$, desvio padrão e coeficiente de variação de fabricação dos emissores avaliados

\begin{tabular}{ccccc}
\hline Cor do Bocal $(\mathrm{mm})$ & $\begin{array}{c}\text { Pressão } \\
(\mathrm{kPa})\end{array}$ & $\begin{array}{c}\text { Vazão } \\
\left(\mathrm{L} \mathrm{h}^{-1}\right)\end{array}$ & Desvio Padrão & $\begin{array}{c}\text { CVf } \\
(\%)\end{array}$ \\
\hline \multirow{6}{*}{ Grafite } & 50 & 24.39 & 0,26 & 1,09 \\
& 100 & 30.21 & 0,60 & 2,00 \\
& 150 & 34.42 & 0,40 & 1,19 \\
& 200 & 38.82 & 0,74 & 1,92 \\
& 250 & 42.20 & 0,87 & 2,08 \\
\hline \multirow{3}{*}{ Laranja } & 50 & 87.20 & 3,91 & 4,49 \\
& 100 & 107.50 & 4,92 & 4,58 \\
& 150 & 124.15 & 6,96 & 5,61 \\
& 200 & 137.47 & 7,68 & 5,59 \\
& 250 & 149,95 & 8,64 & 5,74 \\
\hline
\end{tabular}


O coeficiente de variação de fabricação médio para o bocal de cor grafite foi de $1,66 \%$, sendo classificado como bom por ABNT (1986) e de categoria A por ISO 9260 (1991). Para o bocal de cor laranja, com 5,07\% de variação de fabricação médio, classificado também como bom por ABNT

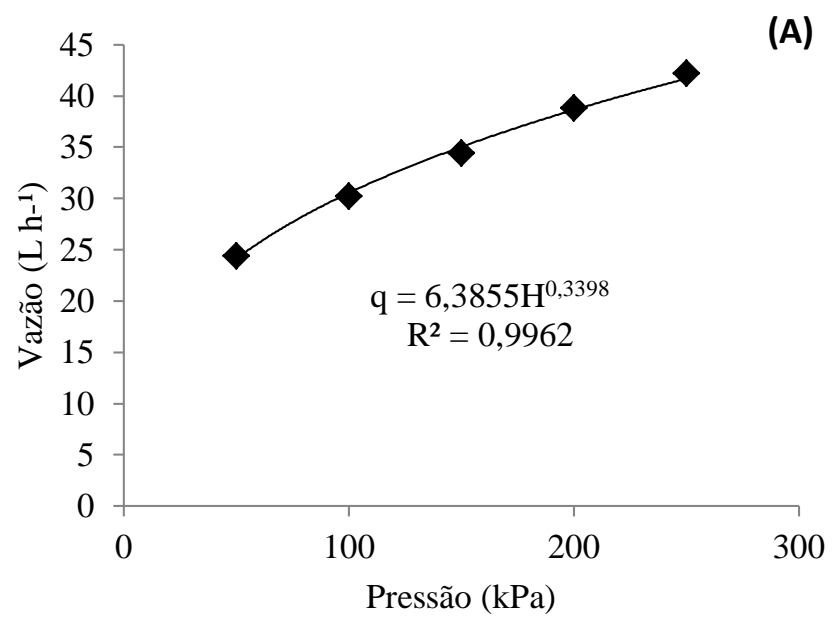

(1986) e de categoria B por ISO 9260 (1991).

A partir da relação dos dados de vazão e pressão determinou-se a equação e curva característica de cada peça. Na Figura 1 são mostradas as curvas características dos microaspersores com os bocais avaliados
(A)

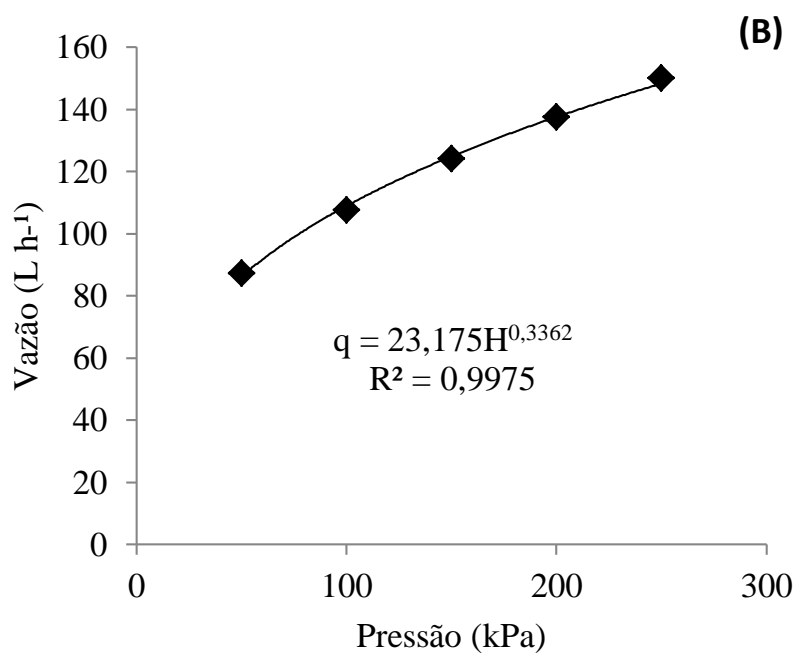

Figura 1. Curva e equação característica vazão-pressão para os emissores do tipo microaspersores, funcionando com os bocais de cor grafite (A) e de cor laranja (B)

As vazões obtidas nos ensaios foram submetidas à análise de correlação, e quando comparadas com as vazões fornecidas pelo fabricante observou-se que os ensaios realizados apresentaram uma pequena tendência a superestimativa, em torno de 8,02 \% em média quando comparadas a reta 1:1 (Figura 2).

Este tipo de emissor é amplamente utilizado em sistemas de irrigação localizada por apresentar pequena variação da vazão e uma alta correlação com os dados fornecidos pelo fabricante, o que aumenta a confiabilidade do produto (SOUZA et al., 2005; MIOLA et al, 2009; CUNHA et al., 2010;). Tais resultados corroboram com Oliveira et al. (2010), quando estudaram a mesma linha de microaspersores, afirmando que estas características podem ter sido proporcionadas por um grande controle de qualidade no processo de fabricação, sendo estes os melhores critérios para avaliar a qualidade destas peças 


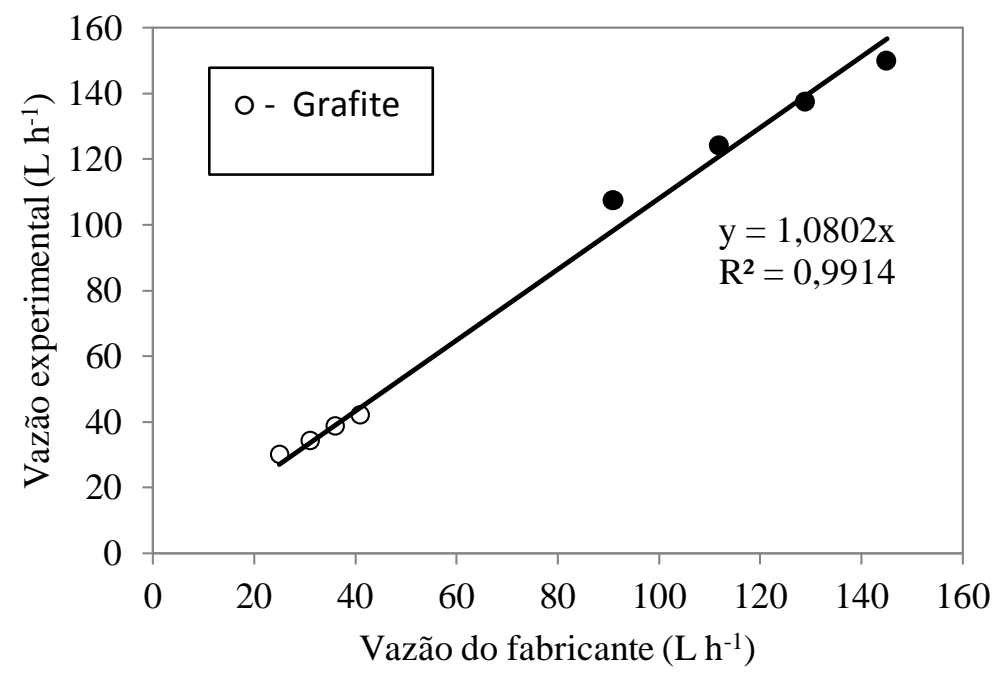

Figura 2. Correlação entre as vazões do fabricante e as obtidas nos ensaios.

\section{Perfil de distribuição de água}

Observando os resultados obtidos, constatou-se um aumento no diâmetro molhado de acordo com o aumento da pressão (50, 150 e $250 \mathrm{kPa})$. Quando comparados com os dados fornecidos pelo fabricante verifica-se que, com uma pressão de serviço de $150 \mathrm{kPa}$, houve um aumento de 9 e 11\% no diâmetro molhado para os bocais grafite e laranja, respectivamente (Tabela 4).

Tabela 4. Diâmetro molhado médio para os bocais analisados.

\section{Diâmetro molhado (m)}

Bocal

Pressão $(\mathrm{kPa})$

\begin{tabular}{ccccc}
\cline { 2 - 5 } & 50 & $150^{*}$ & 150 & 250 \\
\hline Grafite & 4,2 & 5,5 & 6,0 & 6,6 \\
Laranja & 7,2 & 7,0 & 7,8 & 9,0 \\
\hline
\end{tabular}

*Diâmetro fornecido pelo fabricante.

Na Figura 3 são apresentados os perfis de distribuição dos microaspersores avaliados. Pode-se observar uma variação decrescente na precipitação com o aumento da distância em todas as pressões avaliadas. Observando as figuras A1 e A2 nota-se que existe uma região nas proximidades do emissor com baixa precipitação, comportamento este indesejável, pois contribui para a obtenção de baixos valores dos coeficientes de uniformidade quando se trabalha com uma pressão de serviço de $50 \mathrm{kPa}$. 

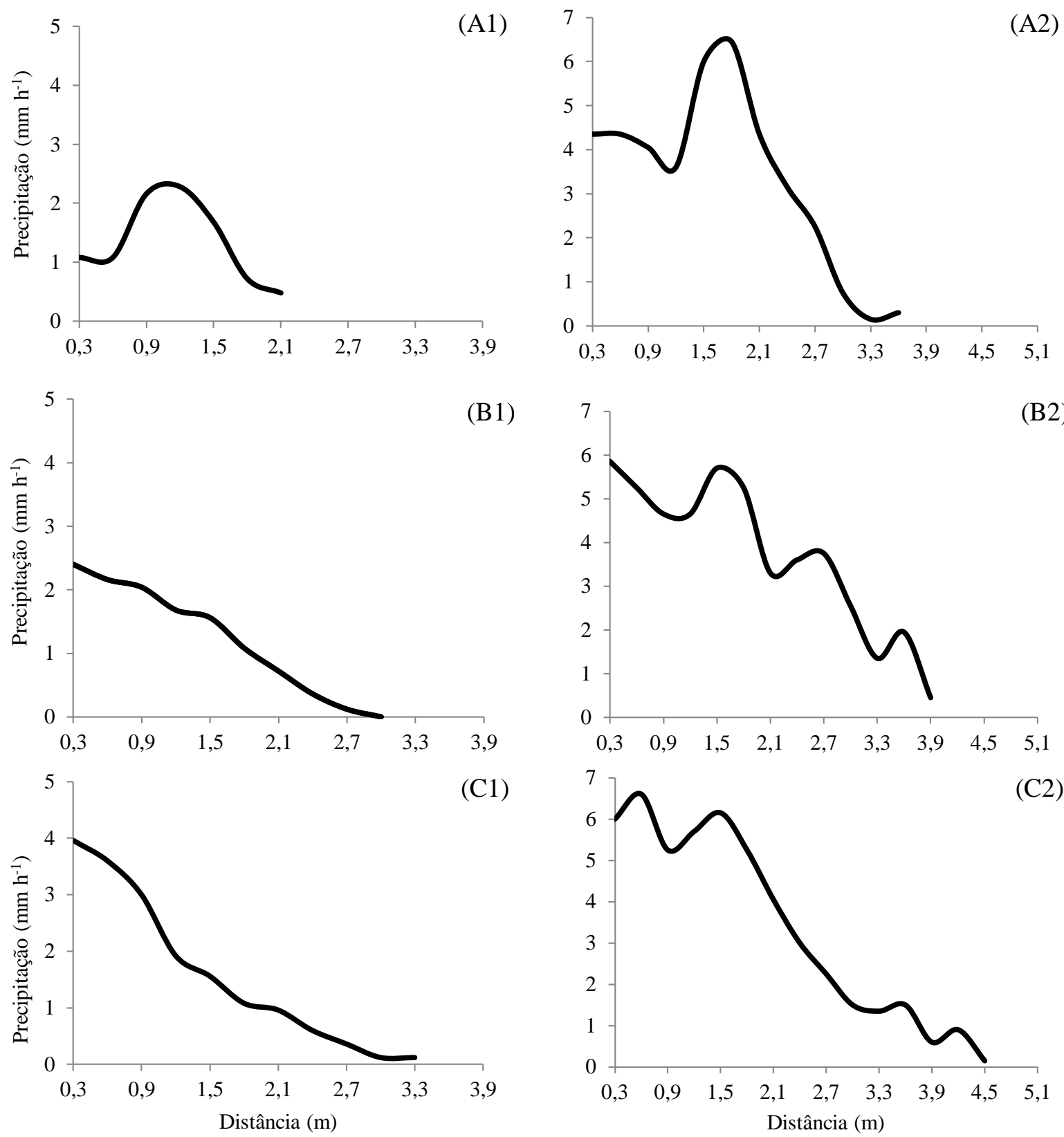

Figura 3. Perfil de distribuição de água dos microaspersores multifuncionais com $50 \mathrm{kPa}$ nos bocais grafite (A1) e laranja (A2), com $150 \mathrm{kPa}$ nos bocais grafite (B1) e laranja (B2), e com $250 \mathrm{kPa}$ nos bocais grafite (C1) e laranja (C2).

Observa-se para todas as pressões estudadas grande amplitude de precipitação entre o emissor e o coletor da extremidade, corroborando Martins et al. (2015) (Figura 3). Quando submetidos a uma pressão de 150 e $250 \mathrm{kPa}$, os microaspersores apresentaram perfis de distribuição com maiores precipitações nas regiões mais próximas do emissor, fazendo-se necessário realizar a sobreposição das lâminas para se obter uma maior uniformidade de distribuição de água.

De acordo com os dados da Tabela 5, para o bocal de cor grafite os maiores valores de CUC foram encontrados quando os microaspersores estavam dispostos a uma sobreposição de 2,1 x 2,1 m, com 86,3\%. Os valores de CUD e CUE foram superiores na sobreposição de 1,2 x 1,2 m, 81,1 e $82,1 \%$, respectivamente, sendo classificado como bom. Já o bocal de cor laranja obteve os maiores CUC $(91,2 \%)$, CUD (88,2\%) e CUE $(90,4 \%)$ na 
Tabela 5. Valores de uniformidade de distribuição para os bocais analisados.

\begin{tabular}{ccccccccccccc}
\hline & \multicolumn{10}{c}{ Sobreposição $(\mathrm{m})$} \\
\cline { 2 - 13 } Bocal & \multicolumn{10}{c}{$1,2 \times 1,2$} & \multicolumn{1}{c}{$1,5 \times 1,5$} & \multicolumn{3}{c}{$2,1 \times 2,1$} & \multicolumn{3}{c}{$3,0 \times 3,0$} \\
& CUC & CUD & CUE & CUC & CUD & CUE & CUC & CUD & CUE & CUC & CUD & CUE \\
\hline Grafite & 84,0 & 81,1 & 82,1 & 82,4 & 75,6 & 79,2 & 86,3 & 79,4 & 81,2 & 76,0 & 67,7 & 72,3 \\
Laranja & 91,2 & 88,2 & 90,4 & 89,2 & 87,3 & 87,3 & 90,0 & 83,9 & 87,3 & 85,4 & 79,0 & 82,2 \\
\hline
\end{tabular}

Assim, observou-se que apenas o microaspersor com o bocal de cor grafite apresentou uma boa uniformidade com uma sobreposição próxima ao diâmetro molhado fornecido pelo fabricante. O bocal de cor laranja apresentou melhor uniformidade em sobreposição muito pequena quando comparada ao diâmetro molhado fornecido pelo fabricante, sendo que a sobreposição 3,0 $\mathrm{x}$ 3,0 m, a qual mais se aproxima dos valores de diâmetro molhado de fábrica, apresentou valores de CUC (85,4\%), CUD (79,0\%) e CUE (82,2\%) com boa classificação segundo Mantovani (2001).

\section{CONCLUSÕES}

As equações características dos bocais avaliados apresentaram coeficientes que caracterizam emissores de baixa variação de vazão, com uma superestimava média de $8,02 \%$, podendo esta representar elevada perda de água no dimensionamento de projetos.

Os emissores com bocais grafite $\mathrm{e}$ laranja apresentaram coeficientes de uniformidade variando de razoável a bom e bom a excelente, respectivamente.

Os emissores avaliados apresentaram melhores coeficientes de distribuição de água quando espaçados em 1,2 x 1,2 m, embora se possa recomendar os espaçamentos de $2,1 \mathrm{x}$ 2,1 para o bocal grafite e 3,0 x 3,0 m para o bocal laranja.

\section{AGRADECIMENTOS}

Os autores agradecem financeiramente ao MCT, ao CNPq, CAPES/PNPD, a FACEPE e ao Instituto Nacional de Ciência e Tecnologia - Salinidade (INCTSal).

\section{REFERÊNCIAS BIBLIOGRÁFICAS}

ÁlVAREZ, J. F. O.; MARTIN-BENITO, J. M. T.; VARELO, J. A. J.; PÉREZ, P. C. Uniformity distribution and its economic effects on irrigation management in semiarid zones. Journal of Irrigation and Drainage Engineering, v.130, p.257-268, 2004.

ASSOCIAÇÃO BRASILEIRA DE NORMAS TÉCNICAS (ABNT). Emissores para sistema de irrigação localizada: avaliação de características operacionais: projeto 12: 02.08.21 São Paulo, 1986. 6 p.

CUNHA, M.D.; CUNHA, M.L.; FREIRE, J.O. Avaliação de desempenho do microaspersor amanco $63 \mathrm{l} \mathrm{h}^{-1}$ em condições de campo. HOLOS, Vol. 5, n.26, p.23-27, 2010.

DETOMINI, E.R.; POWER, B.; FRIZZONE, J.A. Theoretical basis and significance of the variance as a bidimensional variable for design of lateral lines of micro-irrigation. Scientia Agricola, v. 66, p. 563-569, 2009.

FRIZZONE, J. A.; DOURADO NETO, D. Avaliação de sistemas de irrigação. In: MIRANDA, J. H. DE; PIRES, R. C. DE M. (Org.). Irrigação. 1 ed., v. 2, Jaboticabal-SP: FUNEP, 2003, p. 573-651.

ISO - International Organization for Standardization. ISO 9260/1. Agricultural irrigation equipment - emitters - 
specification and test methods,

Switzerland. 1991. 6p.

KELLER, J.; KARMELI, D. Trickle irrigation design. Rain Bird Sprinkler Manufacturing Corporation, 133 p. 1975.

LOUIE, M.; SELKER, J. S. Sprinkler head maintenance effects on water application uniformity. Journal of Irrigation and Drainage Engineering, v.126, p.142-148. 2000.

MACEDO, A. B. M.; GOMES FILHO, R. R.; LIMA, S. C. R. V.; VALNIR JUNIOR, M.; CAVALCANTE JUNIOR, J. A. H.; ARAUJO, H. F. Desempenho hidráulico de um sistema de irrigação por microaspersão utilizando dois tipos de emissores. Revista Brasileira de Agricultura Irrigada, v. 4, p. 82-86, 2010.

MANTOVANI, E. C. AVAliA: Programa de Avaliação da Irrigação por Aspersão e Localizada. Viçosa, MG: UFV, 2001.

MARQUES, P. A. A.; FRIZZONE, J. A.; TEIXEIRA, M. B. O estado da arte da irrigação por gotejamento subsuperficial. Colloquium Agrariae, v. 02, p. 17-31, 2006.

MARTINS, P. E. S.; SILVA, E. R.; SANTANA, V. G.; ARAÚJO NETO, J. A.; ZANINI, J. R. Uniformidade de distribuição de água do microaspersor MC-20. Revista de Ciência Agrária, v. 38, n.1, p.65-70, 2015.

MIOLA, E. C. C.; VIELMO, A. L.; SANTOS, K. L.; SCHONS, R. L.; ROBAINA, A. D.; PEITER, M. X. Curva característica do microaspersor NAANDAN HADAR 7110. Irriga, Botucatu, v. 14, n. 1, p. 95-101, 2009.
OLIVEIRA, E. F.; CRUZ, R. L.; PLETSCH, T. A.; MAZZER, H. R.; SALOMÃO, L. C. Efeito do uso de água cinza sobre a vazão de um microaspersor dinâmico. Irriga, v. 15, p. 258-267, 2010.

PRADO, G.; COLOMBO, A. Composição de perfis radiais de distribuição de água de aspersores. Irriga, v.14, p.41-53, 2009.

RETTORE NETO, O.; FRIZZONE, J.A.; MIRANDA, J.H.; BOTREL, T.A. Perda de carga localizada em emissores não coaxiais integrados a tubos de polietileno. Engenharia Agrícola, v. 29, p. 28-39, 2009.

REZENDE, R.; GONÇALVES, A. C. A.; FREITAS, P. S. L.; FRIZZONE, J. A.; TORMENA, C. A.; BERTONHA, A. Influência da aplicação de água na uniformidade da umidade no perfil do solo. Acta Scientiarum, v.24, n.5, p. 1553-1559, 2002.

SOUZA, J. A. R.; DENÍCULI, W.; BATISTA, R. O.; VAL, J. C. C.; MATOS, A. T. Caracterização hidráulica de microaspersor aplicando água limpa, água residuária de avicultura e de bovinocultura. Engenharia na Agricultura, v.13, n.3, p. 161-172, 2005.

SOUZA, J. A. R. Desempenho de microaspersores operando com água residuárias de avicultura e bovinocultura. 2005. 60f. Dissertação (Mestrado em Engenharia Agrícola) - Universidade Federal de Viçosa, Viçosa. 